\title{
ANTIMICROBIAL EFFICACY OF BIOACTIVE COMPOUNDS OF RARE ENDOPHYTIC ACTINOBACTERIA, Actinoalloteichus cyanogriseus SIR5 (MK793584)
}

\author{
Geetika Wag ${ }^{\bowtie}$, Sunita Datla and Ashwini Kumar Gupta \\ Microbiology Research Laboratory, School of Studies in Life Science, Pt. Ravishankar Shukla \\ University, Raipur-492010, Chhattisgarh, India \\ ${ }^{\square}$ Corresponding Author: geet.mun08@gmail.com
}

\begin{abstract}
To address the problem of antibiotic resistance in pathogens, our research aimed for endophytic actinobacteria, producers of a diverse array of significant bioactive metabolites. Endophytic actinobacteria SIR5 was isolated from roots of Sphaeranthus indicus Linn. and was identified to be Actinoalloteichus cyanogriseus via 16S rRNA sequencing. With the accession number MK793584, the gene sequence was deposited to NCBI. In the current study, a rare actinobacteria Actinoalloteichus cyanogriseus, has been reported as an endophyte for the first time. Both Microbial Type Culture Collection (MTCC) and Clinical Cultures (CC) were used to investigate the antimicrobial property of the bioactive chemicals synthesized by A. cyanogriseus SIR5. A significant zone of inhibitions was recorded against clinical cultures: B. cereus (12.16 $\pm 0.16 \mathrm{~mm})$, Candida albicans $(12.83 \pm 0.44 \mathrm{~mm})$, E. coli $(15.33 \pm 0.33 \mathrm{~mm}), S$. epidermidis $(11.50 \pm 0.28 \mathrm{~mm})$ and MTCC pathogens: B. cereus $(11.16 \pm 0.16 \mathrm{~mm}), B$. subtilis $(13.33 \pm 0.16 \mathrm{~mm})$, P. aeruginosa $(13.33 \pm 0.33 \mathrm{~mm})$, S. epidermidis $(12.33 \pm 0.33 \mathrm{~mm})$. The production of bioactive compound was enhanced by optimization using one factor at a time (OFAT), which was achieved with modified ISP-4 medium (starch - 1\% w/v, $\mathrm{NH}_{4} \mathrm{NO}_{3}-1 \% \mathrm{w} / \mathrm{v}, \mathrm{CaCO}_{3}-2 \mathrm{~g} / \mathrm{l}, \mathrm{K}_{2} \mathrm{HPO}_{4}-1 \mathrm{~g} / 1, \mathrm{MgSO}-1 \mathrm{~g} / 1, \mathrm{NaCl}-$ $1 \mathrm{~g} / \mathrm{l}$, trace solution $-1 \mathrm{ml} / \mathrm{l}$ ) with inoculum size - $13 \%$, incubation period - 16 days, $\mathrm{pH}-8.0$ and temperature $-28^{\circ} \mathrm{C}$. Keywords: Actinoalloteichus cyanogriseus, Bioactive Compounds, Antimicrobial Activity, Endophytic Actinobacteria
\end{abstract}

RASĀYAN J. Chem., Vol. 14, No.3, 2021

\section{INTRODUCTION}

The increased multidrug resistance (MDR) in pathogens as a result of anthropogenic activities in addition to natural processes (through hereditary changes, efflux pump, $\beta$ lactamases, etc.) is alarming for public health and modern medicine. ${ }^{1,2}$ The situation has resulted in reduced effectiveness of approved antibiotics and thus efforts are being made to find efficient and broad-spectrum antibiotics from actinobacteria which are potential producers of diverse metabolites. Since currently available antibiotics are mainly derived from soil actinobacteria, research on endophytic actinobacteria is underway to replace repetitive discovery of known antibiotics. Endophytic actinobacteria are more likely to be involved in the metabolic pathway of the host plant and thus chances of production of some potential novel bioactive metabolites in addition to chemically similar ones are more. ${ }^{3}$ Taxane (taxol), an anticancer compound produced by the plants Taxus brevifolia and Taxus baccata, has also been obtained from its endophyte Micromonospora sp. and Kitasatospora sp. respectively, ${ }^{4}$ possibly evidencing the involvement of both in their metabolism. Thus to ascertain more efficient compounds, recent research has focused on rare endophytic actinobacteria, an underexplored group of microorganisms.

In this study, a rare actinobacteria, Actinoalloteichus cyanogriseus strain SIR5 was procured from the root tissue of medicinal weed Sphaeranthus indicus from Raipur, Chhattisgarh and its antimicrobial activity was observed against MTCC and Clinical pathogens. Previously, A. cyanogriseus has been isolated from the soils of China and the strain was authenticated to be of family Pseudonocardiaceae, based on phylogenetic analysis. ${ }^{5}$ Bioactive alkaloids, caerulomycins and cyanogramide, obtained from marine $A$. cyanogriseus, exhibited significant antibacterial, antifungal, anticancer and antiamoebic activity. ${ }^{6-8}$ 
Chhattisgarh has tremendous diversity and abundance of ethnomedicinal plants, but only a few endophytic actinobacteria have been reported so far. ${ }^{9}$ Therefore, the present research focused on endophytic actinobacteria from this underexplored province.

\section{Materials and Methods}

\section{EXPERIMENTAL}

\section{Isolation of Endophytic Actinobacteria}

Blooming S. indicus plants were collected from the vicinity of Pt. Ravishankar Shukla University, Raipur, Chhattisgarh. The root tissues were processed aseptically within $24 \mathrm{~h}$ of collection following Chandrakar \& Gupta, 2015. ${ }^{10}$ The surface-sterilized root tissues were then inoculated either directly on the growth medium or were macerated and spread on ISP-1, starch casein nitrate agar (SCN agar) and yeast extract malt extract agar media. The growth of other endophytic fungus and fast-growing bacteria was inhibited by the addition of cycloheximide/ nystatin $(50 \mu \mathrm{g} / \mathrm{ml})$ and nalidixic acid $(50 \mu \mathrm{g} / \mathrm{ml})$, respectively to the culture media. The incubation period provided was 21 days at $28^{\circ} \mathrm{C}$.

\section{Morphological Characterization}

The morphological characteristics such as growth pattern, colony nature, pigmentation, aerial and substrate mycelium of A. cyanogriseus were observed on seven different culture media; ISP-3, ISP-4, ISP-5, ISP-6, ISP-7, glucose soybean meal agar (GSMA) and SCN agar. ${ }^{11,12}$ The spore chain morphology was analyzed under the light microscope (Leica DM 1000) and scanning electron microscope (SEM).

\section{S rRNA Gene Sequencing and Phylogenetic Analysis}

Extraction of the genomic DNA and PCR amplification of the strain SIR5 was done using ZR Bacterial DNA Mini Prep kit (Make Zymo Research) and universal primers 27F (AGAGTTTGATCCTGGCTCAG) and 1492R (TACGGYTACCTTGTTACGACTT), respectively. QIAquick Gel Extraction Kit (Qiagen) was utilized for purification of the amplicon and Sanger DNA sequencing method was applied for obtaining the purified product. The analysis of obtained gene sequence was done by Finch TV software version 1.4. The constructed sequences of 16S rRNA gene were compared via BLAST tool in EzBiocloud portal (http://www.ezbiocloud.net/). The neighbor-joining approach was used to create a phylogenetic tree for strain SIR5 by MEGA6 software.

\section{Test Pathogens}

MTCC pathogens: Bacillus cereus (MTCC-430), Bacillus subtilis (MTCC-441), Staphylococcus aureus (MTCC-96), Staphylococcus epidermidis (MTCC-435), Escherichia coli (MTCC-1687), Klebsiella pneumoniae (MTCC-3384), Proteus vulgaris (MTCC-744), Pseudomonas aeruginosa (MTCC-741), Aspergillus niger (MTCC-872) and Candida albicans (MTCC-183) were acquired from Microbial Type Culture Collection and Gene Bank, IMTECH Chandigarh. Clinical cultures (CC) of B. cereus, B. subtilis, $S$. aureus, $S$. epidermidis, E. coli, K. pneumonia, $P$. vulgaris, $P$. aeruginosa, A. niger and $C$. albicans were obtained from Jawaharlal Nehru Medical College, Raipur, Chhattisgarh, India.

\section{Antimicrobial Activity \\ Primary Screening}

A. cyanogriseus SIR5 was screened for its antimicrobial potential against the test pathogens by the crossstreak method..$^{13}$ Growth inhibition was noted as complete inhibition (CI), declined growth (DG) and uninhibited growth (UG).

\section{Secondary Screening}

The secondary screening was performed against test pathogens, using the disc diffusion method. ${ }^{14}$ Briefly, A. cyanogriseus was inoculated and incubated in $50 \mathrm{ml}$ of SCN broth for 14 days at $28^{\circ} \mathrm{C}$ and $150 \mathrm{rpm}$. The sterile disc was then loaded with crude filtrate, placed on the plates inoculated with test cultures and left for incubation at $37^{\circ} \mathrm{C}$ for $24 \mathrm{~h}$. The antimicrobial activity was measured in terms of the diameters of halos generated. Standard antibiotic discs viz., tetracycline $(10 \mu \mathrm{g})$ and chloramphenicol $(10 \mu \mathrm{g})$ were used as control. 
RASĀYAN J. Chem.

Vol. 14 | No. 3 |2048-2055| July - September | 2021

\section{Optimization of Culture Conditions Using One Factor at a Time (OFAT) Method}

Various physical, chemical and nutritional parameters were optimized by the OFAT method by varying a particular parameter and keeping the other factors constant. The most appropriate nutrition medium was selected from different culture media namely; Czapek-Dox broth (CZB), glucose soybean meal broth (GSMB), glycerol asparagine broth (GAB), ISP-4 broth, nutrient broth (NB), oat meal broth (OMB), SCN broth, soybean meal broth (SMB), tyrosine yeast extract broth (TYEB) and yeast extract malt extract broth (YEMEB). Strain SIR5 was inoculated and incubated in $100 \mathrm{ml}$ of each culture medium at $28^{\circ} \mathrm{C}$ for 14 days at $150 \mathrm{rpm}$ and then the antimicrobial activity of each of the culture filtrates was investigated. Various physical parameters affecting the production of antimicrobial compound were analyzed by taking different ranges of particular parameter such as inoculum size $(1,3,5,7,9,11,13,15,17,19 \%)$, incubation period (1- 20 days), $\mathrm{pH}(5.0,6.0,7.2,8.0,9.0)$ and temperature $\left(26,28,30,32,34,36^{\circ} \mathrm{C}\right)$. Different carbon sources (fructose, glucose, glycerol, lactose, maltose, mannitol, sorbitol, starch, sucrose, xylose) were used at $1 \% \mathrm{w} / \mathrm{v}$ in the medium for studying their effect on the production of the antimicrobial compound. Similarly, to observe the effect of nitrogen source, ammonium nitrate, ammonium sulfate, beef extract, malt extract, peptone, potassium nitrate, sodium nitrate, soybean meal, urea and yeast extract were dissolved in the production media at $1 \% \mathrm{w} / \mathrm{v}$. For each optimization set up, $A$. cyanogriseus SIR5 was inoculated and incubated in $50 \mathrm{ml}$ of SCN broth and the bioactive compound production was observed in terms of its antimicrobial efficacy. Besides, dry cell weight $(\mathrm{g} / 100 \mathrm{ml})$ of the culture biomass was also measured for each optimization set up.

\section{Isolation of Endophytic Actinobacteria}

\section{RESULTS AND DISCUSSION}

A gray colored colony of A. cyanogriseus SIR5 was isolated from the root tissue of S. indicus on SCN agar medium. The successful isolation of the endophytic actinobacteria was possible due to the surface sterilization process, which eliminated the epiphytic micro-organisms. To our knowledge, this study is the first to report $A$. cyanogriseus SIR5 as an endophyte. Previously, A. cyanogriseus has been reported from the soil ${ }^{5}$ and marine sediments ${ }^{8}$ of China. Several other diverse genera of rare actinobacteria have been reported as endophytes like; Actinoallomurus, Amnibacterium, Amycolatopsis, Kineococcus, Micromonospora, Nonomuraea, Oerskovia, Promicromonospora and Rhodococcus. ${ }^{15-19}$ Mostly, the isolated free-living actinobacteria belong to the genus Streptomyces, while reports on rare actinobacteria are poor. Many studies indicate the likeliness of plant internal tissues to harbor rare actinobacteria. ${ }^{20}$

\section{Morphological Characterization}

The culture characteristics such as colony nature, growth pattern, pigmentation, aerial and substrate mycelium are one of the classical approaches for the identification of actinobacteria. In this study, various cultural characteristics have been observed (Table-1) in seven different culture media. The fast growth of the strain SIR5 was observed in ISP-4, GSMA and SCN agar media, whereas moderate growth was noticed in ISP-3, ISP-5, ISP-6 and ISP-7 media. Strain SIR5 was found to release soluble and colored pigments in all the used culture media, except in ISP-5. The color and development pattern of aerial and substrate mycelia also varied with culture media. On visualization under the light microscope (Leica DM 1000), the mycelium was found to be branched and fragmented with rod-shaped spores (Fig.-1B). SEM analysis revealed long, wavy spore chain morphology and rod-shaped spores of average size $1.23 \times 0.46$ $\mu \mathrm{m}$ (Fig.-1C and D). Marine-derived Actinoalloteichus species ${ }^{21}$ also revealed similar observations. However, contrasting culture characteristics have been recorded for the strain A. cyanogriseus IFO $14455^{\mathrm{T}}$ isolated from the soil of Yunnan province of China. ${ }^{5}$ The differences in cultural characteristics of type strains might be due to ecological and physiological variations.

\section{Molecular Characterization}

16S rRNA gene sequencing of strain SIR5 revealed 100\% similarity with Actinoalloteichus cyanogriseus DSM 43889 (AUBJ01000042) and thus ascertained the strain to be of the family Pseudonocardiaceae. The phylogenetic tree revealed $99.56 \%$ and $96.59 \%$ similarity of A. cyanogriseus SIR5 to Actinoalloteichus spitiensis RMV-1378 (AGVX02000100) ${ }^{22}$ and Actinoalloteichus hymeniacidonis 
RASĀYAN J. Chem.

Vol. 14 | No. 3 |2048-2055| July - September | 2021

HPA177, respectively while Streptomyces carpaticus NBRC 15390 (AB184641) was put in the outgroup. Further, an accession number MK793584 was received on submission of the gene sequence of strain SIR5 to the NCBI gene bank.

Table 1: Culture Characteristics of Actinoalloteichus cyanogriseus SIR5

\begin{tabular}{l|l|l|l|l}
\hline Medium & Growth and Colony Nature & Aerial mycelium & Substrate mycelium & Pigmentation \\
\hline GSMA & Fast \& powdery & Whitish gray & Black & Bluish black \\
\hline SCN agar & Fast \& powdery & Gray & Black & Bluish black \\
\hline ISP-3 & Moderate \& powdery & Grayish white & Black & Bluish black \\
\hline ISP-4 & Fast \& smooth & White & Black & Bluish black \\
\hline ISP-5 & Moderate \& powdery & Off White & Gray & No soluble pigment \\
\hline ISP-6 & Moderate \& smooth & White & Pink & Light pink \\
\hline ISP-7 & Moderate \& powdery & Cream & Off white & Creamish white \\
\hline & & & & \\
&
\end{tabular}

Fig.-1: Morphology of Actinoalloteichus cyanogriseus SIR5; (A) in ISP-4 Medium, (B) Light Microscopic (Leica DM 1000) image at x1000, (C-D) SEM Micrograph at x5000 Magnification.

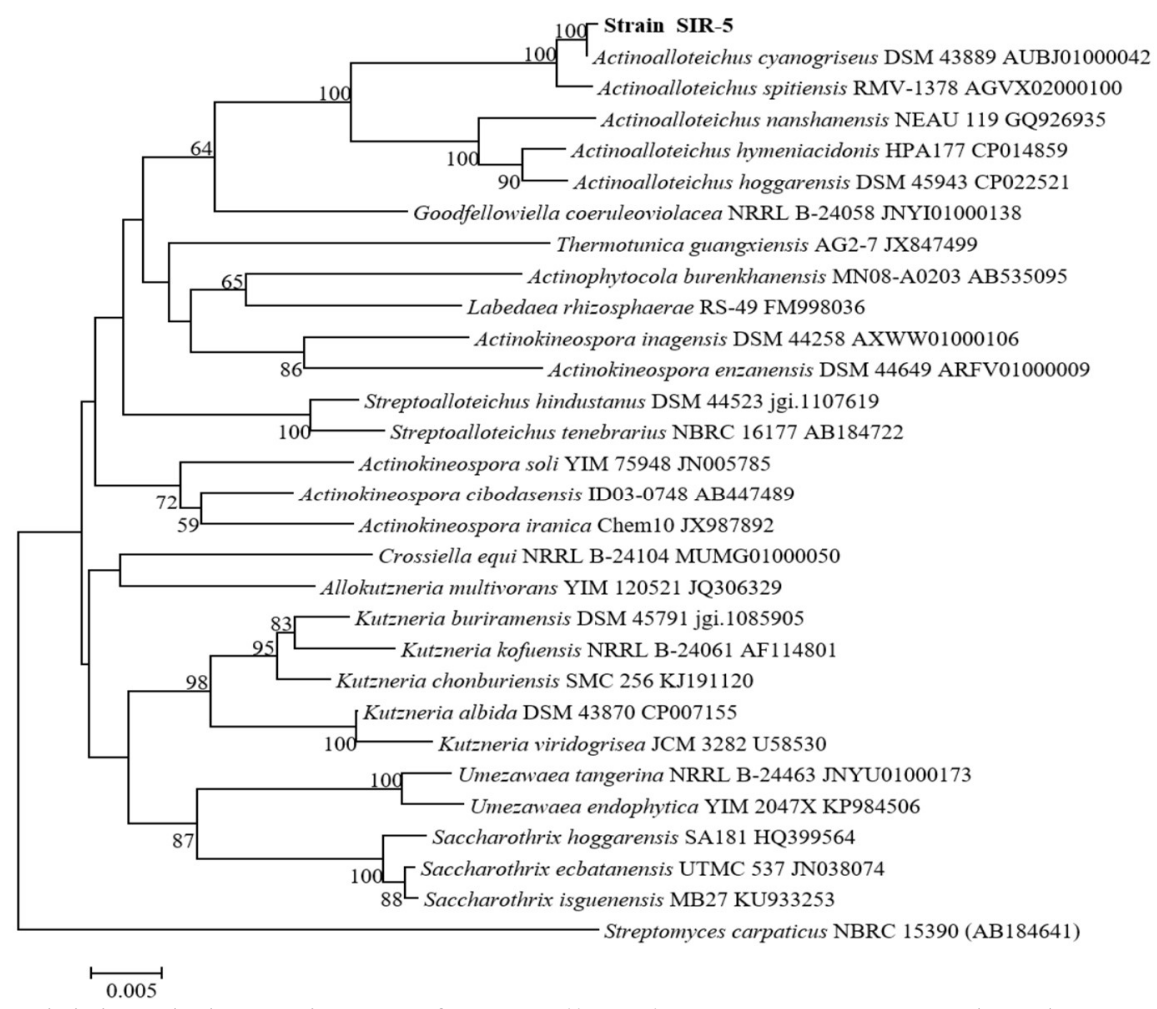

Fig.-2: Neighbor-joining Phylogenetic Tree of Actinoalloteichus cyanogriseus SIR5 based on 16S rRNA Gene Sequence. Values on the Nodes indicate Bootstrap Values, expressed in percentages of 1000 replication.

\section{Antimicrobial Activity \\ Primary Screening}

The antimicrobial activity of strain SIR5 was assessed primarily against 20 pathogens from MTCC and $\mathrm{CC}$, including eight Gram-positive and eight Gram-negative bacteria and four fungal cultures. The results 
RASĀYAN J. Chem.

Vol. 14 | No. 3 |2048-2055| July - September | 2021

(Table-2) showed that all the bacteria except $P$. aeruginosa (CC) were inhibited and significant antimicrobial activity was recorded against $B$. cereus, $S$. aureus, $S$. epidermidis, B. subtilis and E. coli (Fig.-3). However, all the fungal pathogens except $C$. albicans (CC) were found to be resistant. This result supports the hypothesis of the existence of antimicrobial potential in A. cyanogriseus SIR5. Several investigations on the bioactive potential of endophytic actinobacteria indicate that they may be a reservoir of many important pharmaceutical compounds. ${ }^{23-25}$

Table-2: Primary Screening of Antimicrobial Activity of Actinoalloteichus cyanogriseus SIR5

\begin{tabular}{c|c|c|c|c|c|c|c|c|c|c|c}
\hline \multicolumn{2}{c|}{ Pathogens } & $A n$ & $B c$ & $B s$ & $C a$ & $E c$ & $K p$ & $P a$ & $P v$ & $S a$ & $S e$ \\
\hline $\begin{array}{c}\text { Antimicrobial } \\
\text { Activity }\end{array}$ & MTCC & UG & DG & DG & UG & CI & DG & DG & DG & DG & DG \\
\cline { 2 - 25 } & UG & CI & CI & DG & CI & DG & UG & DG & DG & CI \\
\hline
\end{tabular}

An - A. niger, Bc - B. cereus, Bs - B. subtilis, Ca - C. albicans, Ec - E. coli, Kp - K. pneumoniae, Pa - P. aeruginosa, $P v$ - P. vulgaris, $S a-S$. aureus, Se - S. epidermidis, DG - declined growth, UG - uninhibited growth, CI - complete inhibition
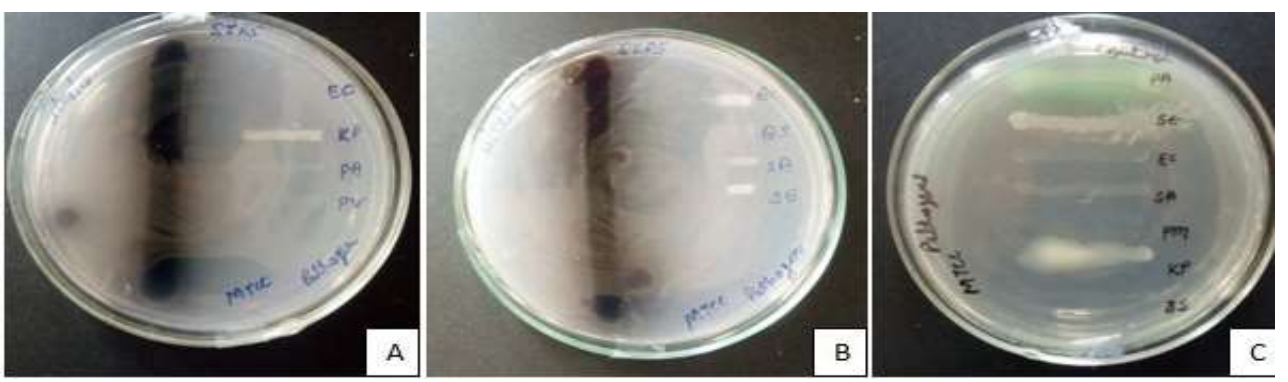

Fig.-3: Antimicrobial Activity of Actinoalloteichus cyanogriseus SIR5 against the pathogens; (A-B) Test Plate, (C) Control

\section{Secondary Screening}

The crude filtrate of $A$. cyanogriseus SIR5 was tested for antimicrobial activity against the pathogens inhibited in primary screening. The zone of inhibition (ZOI) was recorded (Fig.-4) as 15.33 \pm 0.33 , $13.33 \pm 0.16,13.33 \pm 0.33,12.33 \pm 0.33,12.83 \pm 0.44,12.16 \pm 0.16,11.50 \pm 0.28$ and $11.16 \pm 0.16 \mathrm{~mm}$ against $E$. coli (CC), B. subtilis (MTCC), P. aeruginosa (MTCC), S. epidermidis (MTCC), Candida albicans (CC), B. cereus (CC), S. epidermidis (CC) and B. cereus (MTCC), respectively. However, the filtrate produced less effect against $S$. aureus (CC), P. vulgaris (CC) and $K$. pneumoniae (MTCC) with ZOI recorded as $9.16 \pm 0.16,9.83 \pm 0.33$ and $9.33 \pm 0.33 \mathrm{~mm}$, respectively. The overall findings suggested that Gram-negative bacteria were more resistant than Gram-positive bacteria, except E. coli (CC) and P. aeruginosa (MTCC). The endophytic actinobacteria isolated from medicinal herbs also exhibited similar antimicrobial activity. ${ }^{10}$ The susceptibility to antimicrobial compounds may be explained in terms of the cell wall complexity. Vulnerability of Gram-positive bacteria to antimicrobial compounds is attributed to the existence of a single peptidoglycan layer in their cell wall, whereas the existence of lipopolysaccharide (LPS) layer over the peptidoglycan layer in Gram-negative bacteria makes them less susceptible. ${ }^{26}$

\section{Optimization of Culture Conditions}

In this study, $A$. cyanogriseus SIR5 was grown with variations in nutritional, physical and chemical parameters and the antimicrobial activity, in terms of bioactive compound production efficacy, was recorded against the pathogens. The maximum inhibition zone against the pathogens indicated higher antimicrobial compound production. Minimum and maximum production was observed in OMB and ISP4 medium, respectively (Fig.-5A). Optimization of inoculum size (Fig.-5B) revealed a gradual increase in metabolite production from $1-13 \%$ of inoculum size and thereafter decline was observed. The dry cell biomass of the strain also increased accordingly. The production of antimicrobial compound started from the $6^{\text {th }}$ day of incubation and maximum yield was noticed on the $16^{\text {th }}$ day (Fig.-5C). The optimum $\mathrm{pH}$ of the medium was found to be 8.0 by OFAT (Fig.-5D), while at $\mathrm{pH} \mathrm{5,6}$ and 9, strain SIR5 was unable to produce the antimicrobial compound. Optimum temperature was determined by growing the strain at 
RASĀYAN J. Chem.

Vol. 14 | No. 3 |2048-2055| July - September | 2021

various temperatures, such as $26,28,30,32,34$ and $36^{\circ} \mathrm{C}$. Maximum and minimum production of the compound was noticed at $28^{\circ} \mathrm{C}$ and $34^{\circ} \mathrm{C}$, respectively (Fig.-5E).

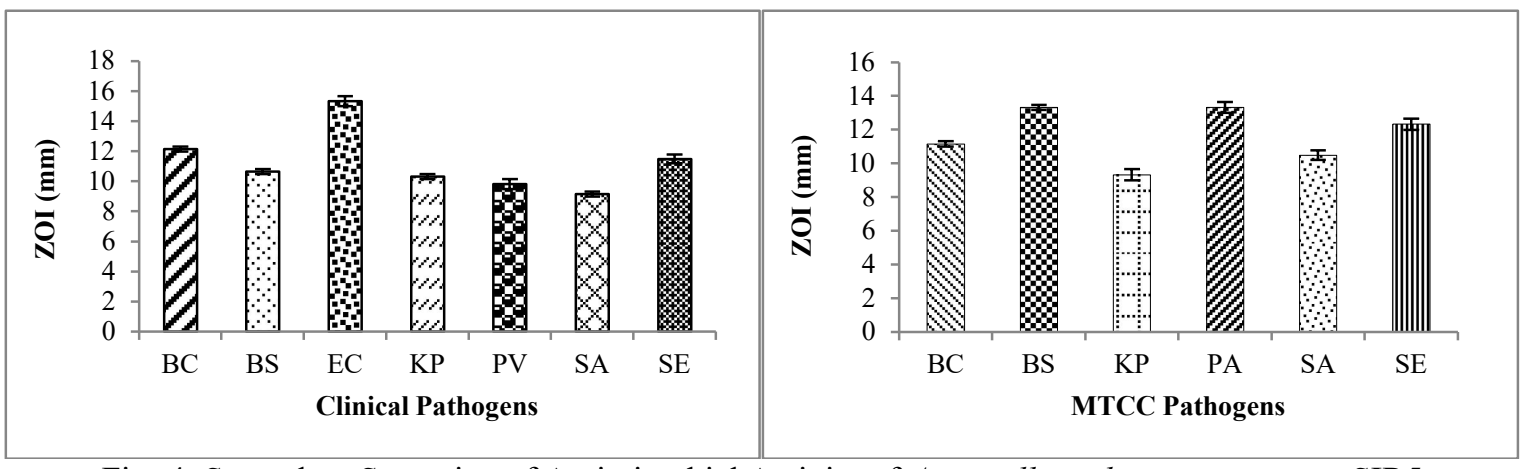

Fig.-4: Secondary Screening of Antimicrobial Activity of Actinoalloteichus cyanogriseus SIR5
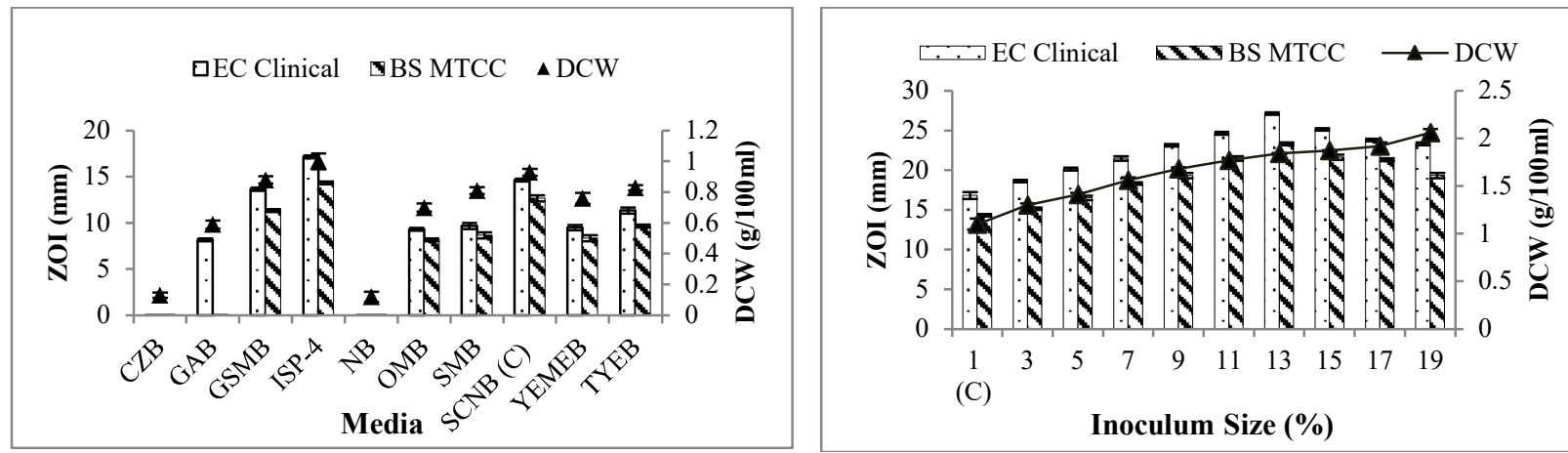

(A)

(B)

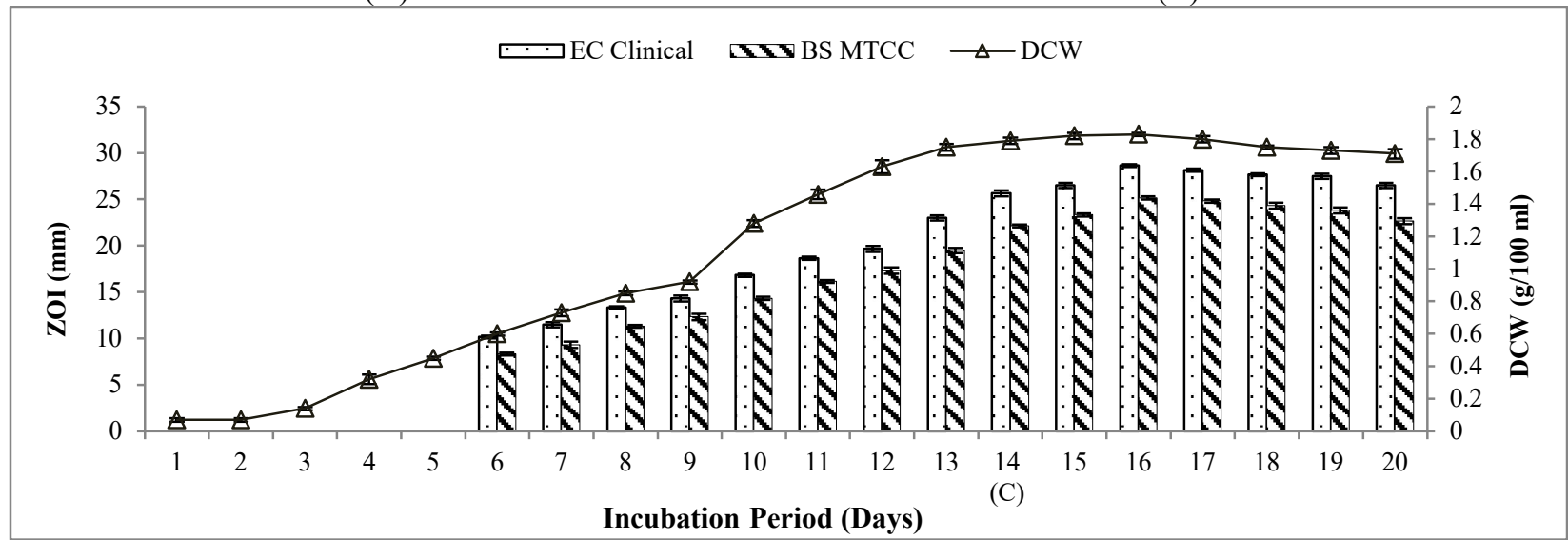

(C)

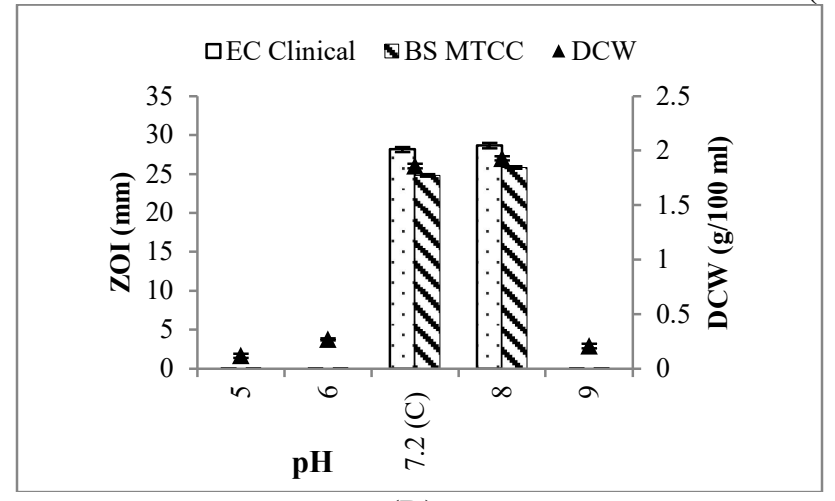

(D)

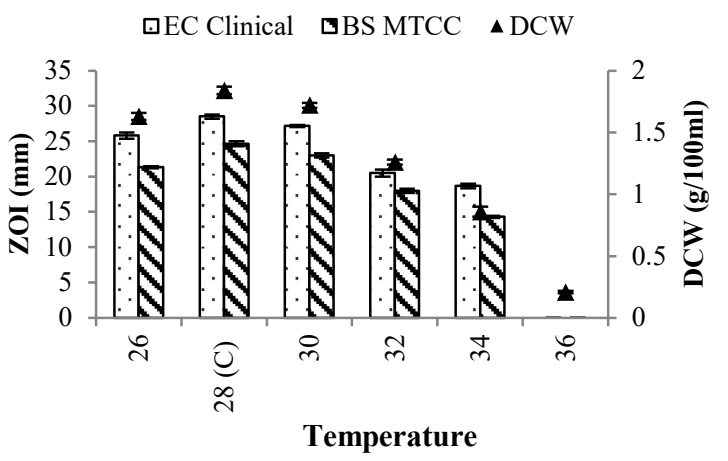

(E)

BIOACTIVE COMPOUNDS 
RASĀYAN J. Chem.

Vol. 14 | No. 3 |2048-2055| July - September | 2021

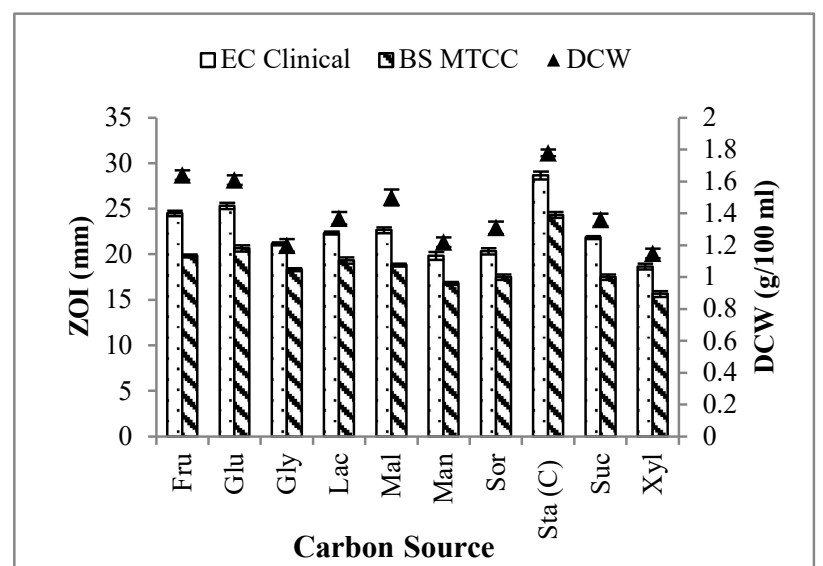

(F)

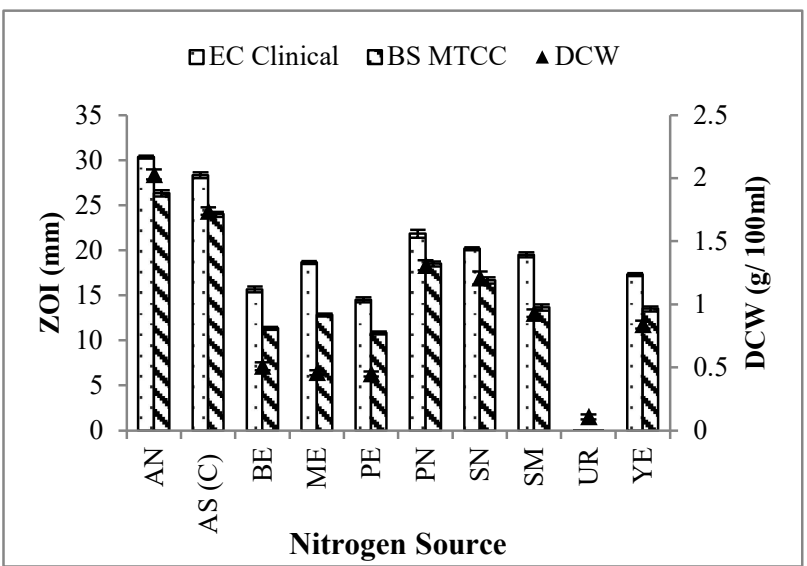

(G)

Fig.-5: Effect of Nutritional, Physical and Chemical Parameters on Antimicrobial Activity of Actinoalloteichus cyanogriseus SIR5 against E. coli (CC) and B. subtilis (MTCC); A - Culture media, B - Inoculum size, C Incubation period, D - pH, E - Temperature, F - Carbon sources, G - Nitrogen sources.

Medium containing starch and ammonium nitrate as carbon and nitrogen source respectively were found to be best for compound production (Fig.-5F and G) while xylose and peptone containing medium showed minimum production. When cultured in optimized conditions, the antimicrobial compound production enhanced significantly which was visible by increased ZOI from $14.66 \pm 0.16$ to $30.33 \pm 0.16$ $\mathrm{mm}$ against $E$. coli (CC) and $12.66 \pm 0.33$ to $26.33 \pm 0.33 \mathrm{~mm}$ against $B$. cereus (MTCC). These results are in agreement with various other studies, which suggest that after optimization, the efficiency of bioactive compounds increases significantly and the optimum fermentation conditions for metabolite production may vary with strains, species, and genus of actinobacteria. ${ }^{27-29}$

\section{CONCLUSION}

Actinobacteria have contributed significantly to the discovery of efficient antibiotics to combat multidrugresistant bacteria. The current study suggested that special attention to rare endophytic actinobacteria may uncover many diverse and broad-spectrum antibiotics, since they are an underexplored group. Our findings showed that A. cyanogriseus SIR5 has potent antimicrobial activity against a wide range of pathogenic bacteria and also ensures the existence of some rare endophytic actinobacteria in Chhattisgarh as it has huge therapeutic floral diversity.

\section{ACKNOWLEDGEMENT}

The authors thank the administrators of Pt. Ravishankar Shukla University, Raipur, Chhattisgarh, for the research facilities provided. The research was financially supported by the CCOST, Raipur and DRSSAP, UGC, New Delhi. The fellowship award to one of us (GW) from PRSU Raipur is duly acknowledged. The thanks are also due to MTCC, Chandigarh, \& JNMC, Raipur for providing cultures and Sophisticated Test and Instrumentation Center (STIC) Cochin, Kerala, India for SEM facility.

\section{REFERENCES}

1. G. L. Conn, V. Bavro, C. Davies, Frontiers in Molecular Biosciences, 6, 71(2019), https://doi.org/10.3389/fmolb.2019.00071

2. World Health Organization, High Level of Antibiotic Resistance Found Worldwide, New Data Shows, World Health Organization, Geneva, Switzerland (2018).

3. L. M. Castronovo, A. Vassallo, A. Mengoni, E. Miceli, P. Bogani, F. Firenzuoli, R. Fani, V. Maggini, Pathogens, 10, 106(2021), https://doi.org/10.3390/pathogens10020106

4. M. Caruso, A. L. Colombo, L. Fedeli, A. Pavesi, S. Quaroni, M. Saracchi, G. Ventrella, Annals of Microbiology, 50, 3(2000).

5. T. Tamura, L. Zhiheng, Z. Yamei, K. Hatano, International Journal of Systematic and Evolutionary Microbiology, 50, 1435(2000), https://doi.org/10.1099/00207713-50-4-1435 
RASĀYAN J. Chem.

Vol. 14 | No. 3 |2048-2055| July - September | 2021

6. P. Fu, S. Wang, K. Hong, X. Li, P. Liu, Y. Wang, W. Zhu, Journal of Natural Products, 74, 1751(2011), https://doi.org/10.1021/np200258h

7. P. Fu, F. Kong, X. Li, Y. Wang, W. Zhu, Organic Letters, 16, 3708(2014), https://doi.org/10.1021/o1501523d

8. Y. Zhu, P. Fu, Q. Lin, G. Zhang, H. Zhang, S. Li, C. Zhang, Organic Letters, 14, 2666(2012), https://doi.org/10.1021/ol300589r

9. S. Chandrakar, A. K. Gupta, Probiotics and Antimicrobial proteins, 11, 1055(2018), https://doi.org/10.1007/s12602-018-9451-6

10. S. Chandrakar, A. K. Gupta, Proceedings of the National Academy of Sciences. India Section B, 87, 905(2017), https://doi.org/10.1007/s40011-015-0668-9

11. Q. Li, X. Chen, Y. Jiang, C. Jiang, Morphological identification of actinobacteria, ActinobacteriaBasics and Biotechnological Applications, InTech Open, Rijeka, Croatia, 59(2016) https://doi.org/10.5772/61461

12. E. B. Shirling, D. Gottlieb, International Journal of Systematic and Evolutionary Microbiology, 16, 313(1966).

13. B. Pandey, P. Ghimire, V. P. Agrawal, Journal of Biological Sciences, 23, 44(2004).

14. M. Balouiri, M. Sadiki, S. K. Ibnsouda, Journal of Pharmaceutical Analysis, 6, 71(2016), https://doi.org/10.1016/j.jpha.2015.11.005

15. C. A. Bascom-Slack, C. Ma, E. Moore, B. Babbs, K. Fenn, J. S. Greene, B. D. Hann, J. Keehner, E. G. Kelley-Swift, V. Kembaiyan, S. J. Lee, P. Li, D.Y. Light, E. H. Lin, M. A. Schorn, D. Vekhter, L. A. Boulanger, W. M. Hess, P.N. Vargas, G. A. Strobel, S. A. Strobel, Microbial Ecology, 58, 374(2009), https://doi.org/10.1007/s00248-009-9494-Z

16. G. K. Bian, Z. Z. Feng, S. Qin, K. Xing, Z. Wang, C. L. Cao, C. H. Liu, C. C. Dai, J. H. Jiang, Antonie van Leeuwenhoek, 102, 621(2012), https://doi.org/10.1007/s10482-012-9757-4

17. C. Li, H. Wang, P. Jin, W. Zheng, L. Chu, C. Liu, X. Wang, Antonie van Leeuwenhoek, 108, 453(2015), https://doi.org/10.1007/s10482-015-0498-Z

18. I. Kim, G. Chhetri, J. Kim, T. Seo, Antonie van Leeuwenhoek, 112, 1731(2019), https://doi.org/10.1007/s10482-019-01302-7

19. K. Zhao, P. Penttinen, T. Guan, J. Xiao, Q. Chen, J. Xu, K. Lindstrom, L. Zhang, X. Zhang, G. A. Strobel, Current Microbiology, 62, 182(2011), https://doi.org/10.1007/s00284-010-9685-3

20. A. S. Azman, I. Othman, S. S. Velu, K. G. Chan, L. H. Lee, Frontiers in Microbiology, 6, 856(2015), https://doi.org/10.3389/fmicb.2015.00856

21. P. A. Jose, S. R. D. Jebakumar, Indian Journal of Geo-Marine Sciences, 44, 428(2015).

22. A. K. Singla, Thesis, CSIR-IMTECH, Chandigarh/Jawaharlal Nehru University, New Delhi, India, (2004).

23. N. Bunbamrung, C. Intaraudom, A. Dramae, C. Thawai, S. Tadtong, P. Auncharoen, P. $\begin{array}{lll}\text { Pittayakhajonwut, } & \text { Phytochemistry, } & \text { 172, }\end{array}$ https://doi.org/10.1016/j.phytochem.2020.112275

24. S. Qin, J. Li, H. H. Chen, G. Z. Zhao, W. Y. Zhu, C. L. Jiang, L. H. Xu, W. J. Li, Applied and Environmental Microbiology, 75, 6176(2009), https://doi.org/10.1128/AEM.01034-09

25. S. Qin, K. Xing, J. H. Jiang, L. H. Xu, W. J. Li, Applied Microbiology and Biotechnology, 89, 457(2011), https://doi.org/10.1007/s00253-010-2923-6

26. D. W. Green, Expert Opinion on Therapeutic Targets, 6, 1(2002), https://doi.org/10.1517/14728222.6.1.1

27. G. Rajivgandhi, R. Vijayan, M. Kannan, M. Santhanakrishnan, N. Manoharan, Bioactive Materials, 1, 140(2016), https://doi.org/10.1016/j.bioactmat.2016.11.002

28. R. Ranjan, V. Jadeja, Journal of Pharmaceutical Analysis, 7, 343(2017), https://doi.org/10.1016/j.jpha.2017.05.001

29. R. Balachandar, N. Karmegam, R. Subbaiya, P. Boomi, D. Karthik, M. Saravanan, Biocatalysis and Agricultural Biotechnology, 20, 101186(2019), https://doi.org/10.1016/j.bcab.2019.101186

[RJC-6432/2021] 